\title{
Arnaud Passalacqua, L'autobus et Paris. Histoire de mobilités
}

Paris, Économica, 2011, 268 p.

Dominique Larroque

\section{OpenEdition}

Journals

Édition électronique

URL : http://journals.openedition.org/dht/1860

DOI : $10.4000 /$ dht. 1860

ISSN : $1775-4194$

Éditeur :

Centre d'histoire des techniques et de l'environnement du Cnam (CDHTE-Cnam), Société des élèves du CDHTE-Cnam

Édition imprimée

Date de publication : 1 décembre 2011

Pagination : 242-244

ISBN : 978-2-9530779-7-1

ISSN : 0417-8726

\section{Référence électronique}

Dominique Larroque, «Arnaud Passalacqua, L'autobus et Paris. Histoire de mobilités », Documents pour l'histoire des techniques [En ligne], 20 | 2e semestre 2011, mis en ligne le 21 septembre 2012, consulté le 22 septembre 2020. URL : http://journals.openedition.org/dht/1860 ; DOI : https://doi.org/ $10.4000 /$ dht. 1860

Ce document a été généré automatiquement le 22 septembre 2020

(c) Tous droits réservés 


\section{Arnaud Passalacqua, L'autobus et Paris. Histoire de mobilités}

Paris, Économica, 2011, 268 p.

Dominique Larroque

\section{RÉFÉRENCE}

Arnaud Passalacqua, L'autobus et Paris. Histoire de mobilités, Paris, Économica, 2011, $268 \mathrm{p}$. 
1 Hors du cercle des spécialistes des transports, il peut paraître surprenant de s'intéresser à l'autobus, avertit d'emblée Arnaud Passalacqua dans l'introduction de son ouvrage, de s'intéresser surtout à son histoire, "éphémère par nécessité ". L'évocation de l'autobus, en effet, renvoie plutôt l'image d'un objet d'une faible technicité ; dans Paris, la configuration du réseau est difficilement identifiable par les citadins; enfin il est réputé être et avoir été sans influence sur l'urbanisme contrairement à ses concurrents du

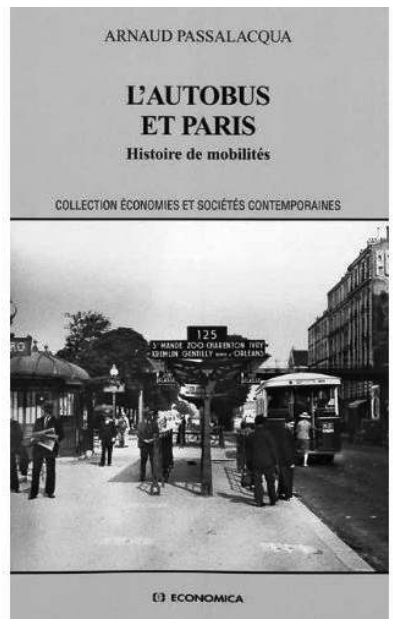
secteur ferroviaire (métro, tramway, chemins de fer). Ainsi l'autobus fut-il généralement perçu «un peu comme une anecdote " ce qui explique au demeurant la place relativement modeste que la bibliographie consacrée aux transports lui a réservée jusqu'à présent. Dès lors, à quoi bon consacrer un ouvrage - quintessence qui plus est d'une thèse - à un objet en apparence anodin ? En fait, Arnaud Passalacqua nous explique que "paradoxalement » il ne tient pas à réhabiliter l'objet de son étude, mais précisément à tenter de comprendre comment celui-ci, l'un des systèmes les plus importants de l'agglomération parisienne, a - parmi ses principales caractéristiques - celle « de passer le plus souvent inaperçu ». Cette entrée en matière annonce l'école de pensée dans laquelle s'inscrit l'ouvrage. Ni - cela va sans dire - l'histoire monographique érudite centrée sur aspect mécanique, matériel des véhicules; ni, l'angle de vue du gestionnaire, de l'exploitant, de l'organisation et des résultats. Mais une démarche qui appréhende l'autobus comme une construction sociale; une démarche qui, sous des angles multiples, veut saisir la complexité des interrelations entre le véhicule urbain et son environnement, c'est-àdire l'espace public, que l'auteur précise entendre de la « voirie aux esprits ».

2 L'ouvrage se divise en deux parties comportant ensemble 6 chapitres. La première partie aborde l'histoire des autobus parisiens sous un angle essentiellement chronologique ; la seconde, en propose une analyse transversale thématique. La période concernée s'inscrit dans la longue durée, de l'apparition de l'omnibus à traction animale à la fin des années 1970. Le premier chapitre se focalise sur une seule ligne, et pas n'importe laquelle : celle des Grands Boulevards, reliant La Madeleine à la Bastille, "idéal-type de l'espace public parisien ». Sous l'angle de cette "microhistoire ", préalable à une approche plus globale des transports parisiens, l'auteur donne à voir au plus près les imbrications entre l'autobus, le contexte urbain et les images qui tour à tour se sont construites autour de la ligne. L'investigation porte sur l'évolution des matériels (de la motorisation d'avant la première Guerre au remplacement en 1993 du bus à plate-forme par des autobus courants), la tarification, les voyageurs, la création des couloirs réservés ... ; mais aussi sur l'inscription de la ligne dans l'espace visuel, sonore et olfactif du boulevard; mais encore sur les impressions des chroniqueurs, romanciers et artistes de l'époque; enfin l'auteur note, pour y revenir dans les chapitres thématiques, le rôle des innovations qui contribuent à faire et défaire les images et les mythes. 
3 Les trois chapitres suivants déroulent une chronique cette fois-ci générale des autobus dans Paris. Le premier des trois débute au début des années 1900, un moment fort dans l'histoire de l'offre de transports dans la capitale. Elle voit simultanément la naissance du métro, la modernisation et l'extension des réseaux de tramways, la venue de l'automobile, la motorisation des omnibus. On traverse ensuite la Grande Guerre durant laquelle les véhicules automobiles démontrent leur efficacité. Le conflit crée également les conditions d'une importante refonte des statuts du système des transports parisiens. Ce chapitre englobe les années 1930, où surgissent de nouveaux défis : la massification de la demande et, avec la croissance du parc automobile parisien, la congestion urbaine. Dans ce nouvel environnement où s'exacerbe la concurrence, où chaque mode a dû trouver son domaine d'élection, on sait que le tramway fut la seule victime. Au travers de cette histoire désormais balisée, Arnaud Passalacqua s'attache à suivre sous un angle inédit le réseau d'autobus, ses adaptations successives, les pratiques qui progressivement s'instaurent, notamment une certaine volonté de normalisation des usages ainsi que des pratiques professionnelles des personnels roulants. Suit un chapitre consacré au "tournant la Seconde Guerre mondiale ». Comme le conflit précédent, l'auteur a raison de dire qu'elle laisse une profonde empreinte sur les transports parisiens. Le transport des troupes sur la ligne Maginot, ou la recherche d'énergies alternatives au pétrole réquisitionné, n'effaceront pas au lendemain du conflit l'image désastreuse des autobus instrumentalisés dans la rafle du Vel d'hiv. Le métro au contraire, qui avait assuré l'essentiel des déplacements des citadins durant l'Occupation ainsi que leur protection lors des bombardements, triomphait. Initiée par le gouvernement de Vichy, la subordination de l'autobus au métro, en germe avant même le début du conflit, mais aussi consubstantielle à la nature des deux systèmes, sera confirmée sous l'égide de la jeune RATP. Les «Trente Glorieuses ", abordées dans un quatrième chapitre, s'ouvrent sur un constat d'«enlisement " progressif des autobus parisiens. Entre autres raisons, Arnaud Passalacqua relève bien sûr la congestion urbaine qui, réduisant la vitesse commerciale, vide les autobus et dégrade leur image. L'auteur date de ces années ce qu'il appelle le "décrochage de l'autobus face à l'automobile ", alors qu'auparavant les deux modes appartenaient à un univers commun en opposition avec leurs concurrents ferroviaires. Il attribue également cet enlisement au fait que le réseau tombe alors sous l'influence de techniciens dont l'expérience, héritée de feu la CMP, est avant tout ferroviaire. Il invoque de même le climat de l'époque vouée à la vitesse et à la réussite, qui tranche avec l'image soudainement désuète d'un autobus paralysé dans la circulation et d'un personnel "enrégimenté " et mal payé. L'autobus résiste pourtant, et à partir des années 1970 connait un regain de faveur, notamment au lendemain de la crise pétrolière, mais au prix d'une "métamorphose " nous dit l'auteur. Il y perdra en souplesse de tracé - sa caractéristique fondamentale - en capillarité, en variété de territoires desservis, et donc en identité : l'instauration des couloirs réservés, inspirée du mode ferroviaire, est à cet égard significative.

4 Ce sont là autant d'éléments de réflexion pointés tout au long de cette chronique, sur lesquels Arnaud Passalacqua revient dans ses deux derniers chapitres afin d'en affiner l'analyse. Parmi les multiples thèmes abordés, l'autobus est ainsi revisité à l'aune de la notion de système (technique et culturel). De même entre autres hypothèses, l'auteur développe l'idée que la dynamique propre à chaque mode tient aux relations avec les autres modes évoluant dans un même espace. Il en résulte des formes d'hybridation intermodale, un mode forçant l'autre à adopter ses propres caractéristiques au risque 
d'être « dévoré » par lui. L'auteur avance à ce propos l'image forte d'un «bestiaire urbain » où les actes de " cannibalisme » ne sont pas rares, suivant en cela Alfred Sauvy qui, de son côté, avait employé la notion de «biologie de la route ", lieu de conversions, d'exterminations et d'exploitations d'espèces. Evoquant ce « champ de batailles » que constitue l'espace public, l'auteur dans son introduction avait posé l'hypothèse de travail selon laquelle «la rigidité des systèmes de transport est bien souvent un avantage plus qu'un inconvénient ". Autrement dit l'autobus, dont la souplesse constituait la qualité inhérente et récurrente, l'autobus dépourvu d'un «corpus technique épais ", n'était certes pas le mieux armé par rapport aux modes ferroviaires. Dans l'un des chapitres thématiques, l'auteur nous livre l'issue de cette "bataille »: afin de naviguer dans ce jeu de contraintes multiples, l'autobus a dû adopter des caractéristiques ambivalentes, "rappelant les capacités du caméléon ", mais un "caméléon complexé »: "complexe d'un mode routier vis-à-vis de l'automobile ", " complexe d'un transport collectif vis-à-vis du métro », " complexe d'un objet ancien vis-à-vis de Paris et de la modernité ». Retour également sur le rôle de l'innovation : elle navigue d'un mode à l'autre, les progrès de l'un forçant l'autre à innover, mais encore faut-il que l'image de la modernité passe dans le public. L'auteur termine son ouvrage avec le regard de l'anthropologue. Il nous invite à monter dans l'autobus, évoque le nécessaire travail d'initiation des usagers, notamment « en matière d'hygiène et de pacification des relations sociales ", contribuant à une moralisation plus générale de la société urbaine. Il revient sur l'image de l'autobus, en particulier sur la place selon lui cruciale de la plate-forme ouverte arrière dans sa construction. La plate-forme est l'espace-clé du fonctionnement de l'autobus parisien, l'interface entre l'espace intérieur et l'espace extérieur, l'élément symbolique par excellence comparable au double deckers londonien.

En donnant à lire au travers « d'un objet anodin, des problématiques très larges » peutêtre l'auteur ne sera-t-il pas suivi dans toutes ses interprétations. Il nous livre en tout cas un travail particulièrement stimulant, créatif, nécessaire contrepoint aux visions hyper-techniciennes qui foisonnent dans l'historiographie des transports. Il nous invite à dépasser l'objet pour s'intéresser aux images qu'il véhicule et qui elles-mêmes influent les choix des décideurs. Il nous livre une réflexion argumentée par de nombreuses références aux travaux de sociologues et d'anthropologues de renom, donnant le mode d'emploi d'une histoire qu'il souhaite ouverte à d'autres champs disciplinaires. Enfin, si les concepts évoqués sont d'un abord parfois difficile, l'écriture est claire et la lecture limpide, ce qui n'est pas la moindre qualité de cet ouvrage. A lire donc, non pas seulement par les passionnés de transports, mais - pour sa méthode par la communauté des chercheurs en histoire des techniques.

\section{AUTEURS}

DOMINIQUE LARROQUE

CDHTE-Cnam 